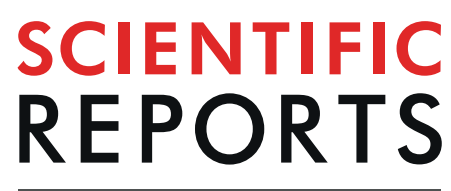

natureresearch

\title{
OPEN Initial Report: A Novel Intraoperative Navigation System for Laparoscopic Liver Resection Using Real-Time Virtual Sonography
}

\author{
Koichiro Sakata $\mathbb{1}^{1,2^{*}}$, Taiki Kijima² \& Osamu Arai $\mathbb{D}^{3}$
}

Recent progress in navigation has revealed problems involving non-rigid registration for hepatic surgery. With the increasing popularity of laparoscopic liver surgery, a new laparoscopic navigation system is necessary. This study involved an in-vitro demonstration of a 3-dimensional printer model and in vivo demonstration in four patients. For the in vitro examination, a position detecting unit attached at $33 \mathrm{~cm}$ and $13 \mathrm{~cm}$ distance conditions from the tip of the electrocautery was examined eight times at the marked points on the liver surface eight times respectively. The differences between the simulation and the authentic dissecting plane were conventionally investigated in vivo. In vitro, the errors of the $33 \mathrm{~cm}$ and $13 \mathrm{~cm}$ distance model were $7.8 \pm 3.5 \mathrm{~mm}$ (mean $\pm S D$ ), and $3.3 \pm 1.0 \mathrm{~mm}$, respectively. The mean differences of the dissection plane were within $10 \mathrm{~mm}$. The potentiality and safety of the novel navigation system was confirmed, although further investigation is recommended.

Recently, liver image constructions using 3-dimensional (3D) simulation software have been utilised in diverse ways to predict the excision lines and anatomical structure of the liver, with variations, and have played important roles in safe and appropriate hepatectomy. Although applications of 3D simulation to real-time navigation systems $^{1}$ are devised variously, and reports on its safety ${ }^{2}$ and usefulness ${ }^{3}$ have been marked, more accurate and reliable navigation systems are still necessary.

In the latest remarkable improvements in laparoscopic operations, especially in the field of hepatectomy, conventional clamping methods ${ }^{4}$ have been commonly used to identify excision domains according to the hepatic portal branches with hilar approaches. Staining methods ${ }^{5}$, ultra-sonographic contrast enhance-methods, and indocyanine green near-infrared imaging methods ${ }^{6}$ are, however, unsuitable because of the limits of laparoscopic procedures.

Therefore, it is expected that a safer and more reliable navigation system using $3 \mathrm{D}$ navigation systems and applying the existing real time virtual sonography (RVS) system will be developed.

\section{Methods}

This study was designed to confirm the accuracy and safety of the new laparoscopic navigation system in vitro, as well as the feasibility and safety of the procedure by measuring the error between the simulation domain and the actual excision domain using the gravity centre of the latter as the reference point in vivo, instead of an anatomical point authentically mentioned.

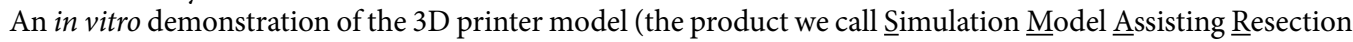
Technique of Liver: SMART Liver, Sony Global Manufacturing \& Operations Corporation, Tokyo, Japan), and in vivo demonstration in four patients with hepatic malignancies (four tumours) were preliminarily investigated in this study between January 2017 and December 2017. Patients who met the following criteria were included in the study: Patients aged $20-80$ years at the time of the agreement acquisition, patients who were scheduled

${ }^{1} J a p a n$ Seafares Relief Association Ekisaikai Moji Hospital 1-3-1 Kiyotaki Mojiku Kitakyushu, Fukuoka, 801-8505, Japan. ${ }^{2}$ JCHO Shimonoseki Medical Centre, 3-3-8 kamishinchi-machi, Shimonoseki, Yamaguchi, 083-231-5811, Japan. ${ }^{3}$ Hitachi, Ltd. Healthcare Business Unit, 3-1-1, Higashikoigakubo, Kokubunji-shi, Tokyo, 185-0014, Japan. *email: atak51ks331007@gmail.com 


\begin{tabular}{|c|c|c|c|c|c|}
\hline \multicolumn{2}{|l|}{ Case } & \multirow{2}{*}{$\begin{array}{l}1 \\
75\end{array}$} & \multirow{2}{*}{$\begin{array}{l}2 \\
77\end{array}$} & \multirow{2}{*}{\begin{tabular}{|l|}
3 \\
78
\end{tabular}} & \multirow{2}{*}{\begin{tabular}{|l|}
4 \\
75
\end{tabular}} \\
\hline Age & y.o. & & & & \\
\hline Sex & $\begin{array}{l}\text { Male: } \mathrm{M} \\
\text { Female: } \mathrm{F}\end{array}$ & M & F & F & M \\
\hline Location of the tumour & Section & VIII & VIII & VIII & VIII \\
\hline Size of the tumour & $\mathrm{mm}$ & 55.66 & 37.26 & 16.28 & 17.53 \\
\hline Performance Status & & 0 & 0 & 0 & 0 \\
\hline ICGR15 & $\%$ & 8.8 & 14.2 & 5.5 & 7.7 \\
\hline $\mathrm{Alb}$ & $\mathrm{g} / \mathrm{dl}$ & 4.2 & 4.3 & 4.8 & 4.4 \\
\hline PT & $\%$ & 93.9 & 82.3 & 72.4 & 76.4 \\
\hline Total bilirubin & $\mathrm{mg} / \mathrm{dl}$ & 0.72 & 0.62 & 0.75 & 0.49 \\
\hline AST & $\mathrm{U} / \mathrm{l}$ & 22 & 26 & 21 & 22 \\
\hline ALT & $\mathrm{U} / \mathrm{l}$ & 29 & 15 & 8 & 24 \\
\hline Platelet & $10^{4} / \mu \mathrm{l}$ & 13.1 & 13.6 & 11.6 & 18.8 \\
\hline Child-Pugh status & & A & A & A & A \\
\hline $\begin{array}{l}\text { Required time for } \\
\text { registration }\end{array}$ & sec. & 150 & 186 & 160 & 136 \\
\hline operative procedure & & sub-sectionectomy & sub-sectionectomy & sub-sectionectomy & sub-sectionectomy \\
\hline $\begin{array}{l}\text { Duration of operation time } \\
\text { (Duration of actual liver } \\
\text { transection time) }\end{array}$ & $\min$. & $356(148)$ & $305(59)$ & $348(69)$ & $263(90)$ \\
\hline Amounts of Blood loss & $\mathrm{ml}$ & 500 & 400 & 400 & 150 \\
\hline Erros Max & $\mathrm{mm}$ & 11 & 5.6 & 22 & 13.3 \\
\hline Mean & $\mathrm{mm}$ & 2 & 1.5 & 8.2 & 4.3 \\
\hline SD & $\mathrm{mm}$ & 3.3 & 1.3 & 6.3 & 3 \\
\hline Surgical margin (distance) & $\mathrm{mm}$ & negative (1) & negative (6) & negative $(0)$ & negative (2) \\
\hline Adverse events after surgery & & $(-)$ & $(-)$ & $\begin{array}{l}\text { post-operative bleeding, } \\
\text { organ SSI, ascites }\end{array}$ & $(-)$ \\
\hline Hospital stay after surgery & days & 20 & 22 & 58 & 26 \\
\hline
\end{tabular}

Table 1. Characteristics of included 4 patients.

for hepatic resection with preoperative images of computed tomography $(\mathrm{CT})$ or magnetic resonance imaging (MRI), patients who had performance status 0 or 1 , and patients who had the following standards of marrow functions: white blood cell count $\geqq 3,500$ cells $/ \mathrm{mm}^{3}$, haemoglobin $\geqq 10.0 \mathrm{~g} / \mathrm{dl}$, and platelet $\geqq 80,000$ cells $/ \mathrm{mm}^{3}$, and the following standards of liver functions total bilirubin level $<2.0 \mathrm{mg} / \mathrm{dl}$, aspartate aminotransferase (AST) level $<100 \mathrm{U} / \mathrm{L}$, alanine aminotransferase (ALT) level $<100 \mathrm{U} / \mathrm{L}$, liver damage A or B.

The patients' characteristics are shown in Table 1. Tumour sizes ranged from $16.3 \mathrm{~mm}$ to $55.7 \mathrm{~mm}$.

Plain CT images of Sony's 3D SMART liver with a $0.8 \mathrm{~mm}$ slice thickness in vitro study/contrast-enhanced CT images with a $0.8 \mathrm{~mm}$ slice thickness of the patients in vitro study were obtained using a multidetector-row CT (SOMATOM Definition S, SIEMENS, Munich, Germany). The anatomical structures of the liver and the portal and hepatic veins were extracted using a 3D simulation software (Synapse Vincent; Fujifilm, Tokyo, Japan). All the reconstructed 3D images and 2D CT images were transferred to the workstation of the RVS system as digital imaging and communication in medicine file data.

An ultrasonography system (HI VISION Ascendus, Hitachi, Tokyo, Japan) with a position detecting unit (EZU-RV3S, Hitachi, Tokyo, Japan) attached to the electrocautery device by surgical tape, was intraoperatively employed to mark the simulated dissecting plane. With the navigation system, operators could refer to intraoperative navigation images displayed on the television monitor side-by-side with corresponding CT and/or MRI images. The system also overlaid a preoperative simulation on the CT image and highlighted the extent of resection so the resection plane could be navigated. Because the system used electromagnetic power in the operation room, the feasibility and safety of the system were investigated along with its validity. The clinical tolerance level of accuracy was ascertained in two steps, an in vitro demonstration of Sony's 3D SMART liver, and in vivo demonstration in four patients with hepatic malignancies (four tumours).

1. In vitro demonstration with Sony's 3D SMART liver, position sensor was set at $33 \mathrm{~cm}$ or $13 \mathrm{~cm}$ distant from the tip of the electrocautery. Registration points were the root of the umbilical portion, the bifurcation of left and right branches, and the base of the eighth portal branch. The error was calculated by measuring the gap of electrocautery tip position on navigating image at the marked point on the liver surface just above the bifurcation of left and right branches eight times, respectively (Fig. 1).

2. An experienced liver surgeon identified the hepatic vessels tributaries as the dominant territory using the $3 \mathrm{D}$ simulation software. In vivo, the $33 \mathrm{~cm}$ distant condition was selected. The tip of the energy device with position sensor was calibrated by touching to the centre of micro-convex probe with another position sensor. Registration points were the same as the in vitro demonstration, and those were set by using a micro-convex probe equipped with a position sensor via a small $7.5 \mathrm{~cm}$ incision below the xiphoid process during laparoscopic surgery according to the method of Takamoto et.al. ${ }^{7}$. 


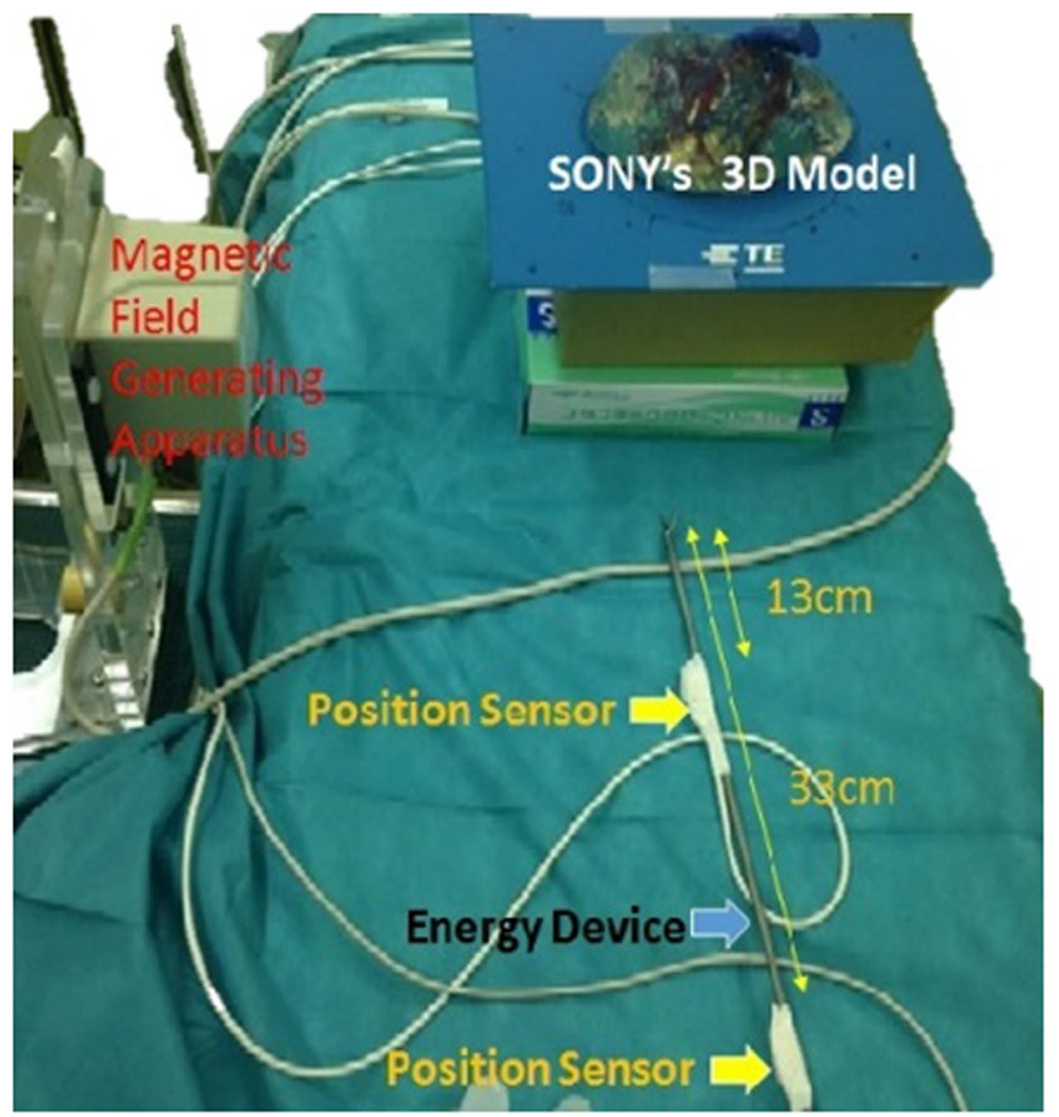

Figure 1. In vitro demonstration with Sony's 3D SMART liver. Distance settings of $33 \mathrm{~cm}$ and $13 \mathrm{~cm}$ away from the tip of the electrocautery were examined at the umbilical portion and the base of the eighth portal branch every eight times, respectively.

The simulation area, based on the three-dimensional construction, was marked by an energy device laparoscopically (Fig. 2a,b). After the J-incision laparotomy, the differences between the simulation plane and the authentic dissecting plane were conventionally investigated after dividing the surrounding ligaments laparoscopically by a staining method via portal branch of section VIII with indocyanine green and ultrasonic enhancement contrast with contrast-enhancement ultrasonography and near-infrared ray vision system (Photo Dynamic Eye ${ }^{\circledR}$ : PDE, HAMAMATSU Photonics, Shizuoka, Japan). And a photograph was taken in front at a height of $15 \mathrm{~cm}$ with a measure attached to the surface of the liver. The actual excision domain was traced using images formed by the software on a computer to calculate the centre of gravity. The differences were measured as a request of errors detected every five degrees of the plane image from the centre in a real excision domain (Fig. 3 ).

Operative-time amounts of bleeding and harmful events according to the Clavien-Dindo classification were searched. Perioperative levels of serum aspartate aminotransferase, alanine aminotransferase, and total bilirubin were measured on the first, third, and seventh days after surgery. Duration of hospital stays were also calculated.

Differences between data of the groups in vitro were evaluated using the Wilcoxon signed rank test. Statistical analysis was performed using $\mathrm{R}$ software. A $\mathrm{p}$ value $<0.05$ was considered statistically significant.

The exploratory study was conducted in accordance with the Declaration of Helsinki and approved by the Institutional Review Board of JCHO Shimonoseki Medical Centre and the ethics committee group of Hitachi (\#201-1). Informed consent was obtained from all the patients.

\section{Results}

During in vitro demonstration, the errors of $33 \mathrm{~cm}$ and $13 \mathrm{~cm}$ distance setting from the tip of the electrocautery revealed the error $7.8 \pm 3.5 \mathrm{~mm}$ (mean \pm Standard Deviation), and $3.3 \pm 1.0 \mathrm{~mm}$, respectively $(\mathrm{P}<0.05)$.

The navigation system was used uneventfully in each operation. Registration time ranged from $136 \mathrm{sec}$. to $186 \mathrm{sec}$ (Table 1). All the four tumours scheduled for resection were detected by the navigation system. The mean differences of the dissection plane between the simulation and conventional procedures were $2.0 \mathrm{~mm}, 1.5 \mathrm{~mm}$, $8.2 \mathrm{~mm}$, and $4.3 \mathrm{~mm}$, respectively, they ranged from $0 \mathrm{~mm}$ to $22 \mathrm{~mm}$ (Table 1 ). No harmful event occurred after surgery and post-operative changes of liver function met the tolerance level. Duration of actual liver transection times were calculated, using the video recording system, as 148, 59, 69 and 90 minutes for case 1, 2, 3, and 4, respectively (Table 1). We spent time for setting up, manipulating, and acquiring data during the surgery than usual because of our unfamiliar with newly developed navigation system. 
a

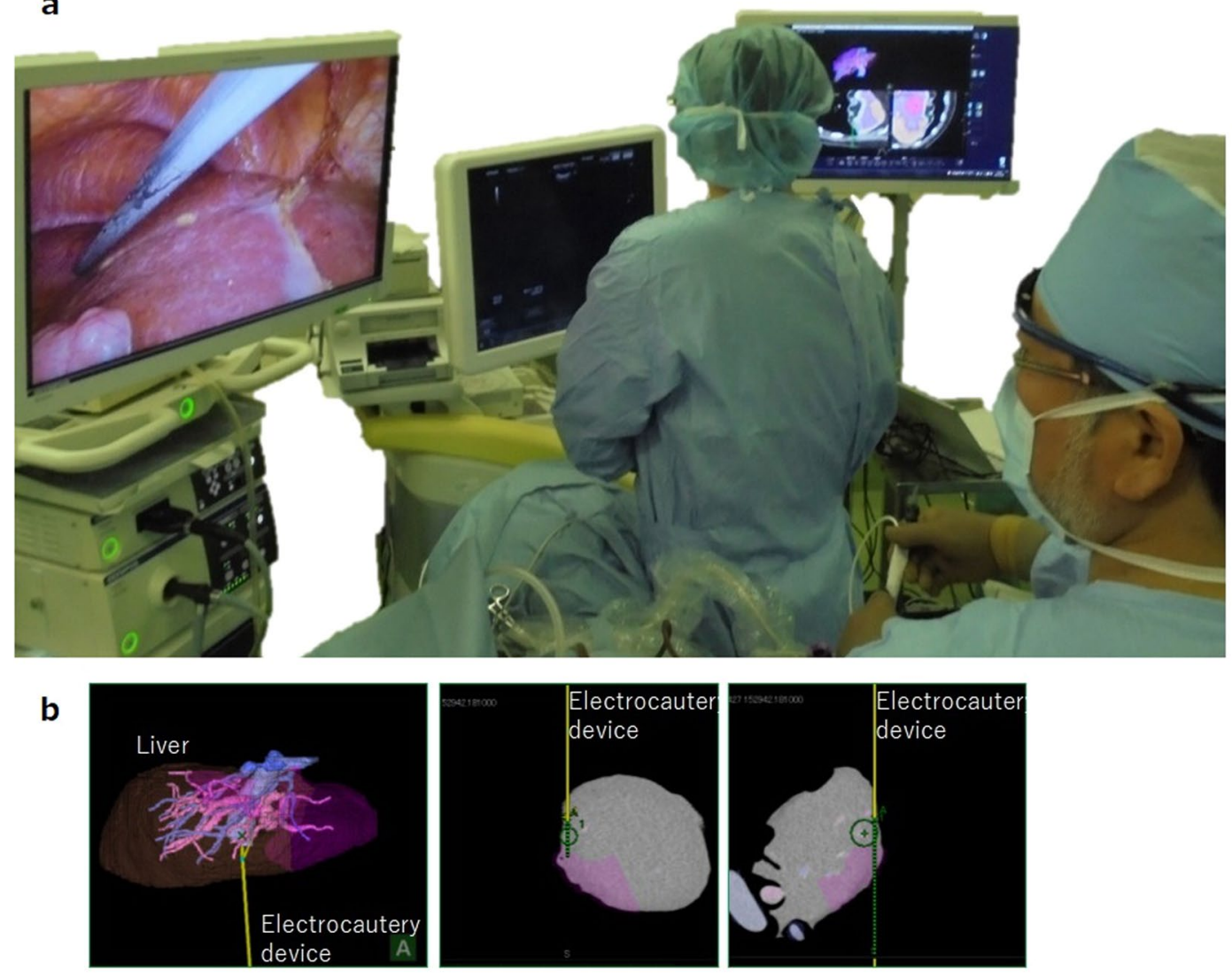

Figure 2. (a) Operators could refer to intraoperative navigation images displayed on the television monitor side-by-side with corresponding CT and/or MRI images. (b) The system overlaid the preoperative simulation on the CT image and highlighted the extent of resection so the resection plane could be navigated.

\section{Discussion}

The anatomic excision of the liver is one of the fundamentally important techniques in hepatic surgery ${ }^{6-10}$.

Despite the confirmation that the correct intersegmental surface is still a technically reliable procedure, a suitable navigation system is still required ${ }^{11-14}$.

This study demonstrated the safety and usefulness of the novel navigation system for laparoscopic hepatic surgery, with parallel displays of images of preoperative CT and/or MRI which synchronised a section using RVS and a $3 \mathrm{D}$ simulation image.

A traditional novel navigation system for open surgery ${ }^{1}$ of liver resection using RVS was reported as useful for the detection of tumours and navigation of the resection plane. Although a reliable system for hepatic surgery requires enough time for the elastic registration between the preoperative imaging data and the intraoperative situation because of hepatic deformation and respiration during surgery, the registration time in our study was feasible. Furthermore, the RVS with the novel automatic registration system ${ }^{7}$ accomplished quick and easy registration as well as acceptable accuracy, which was convenient for our system, with shorter registration time.

In vitro, the difference investigated by the $3 \mathrm{D}$ model was $7.8 \pm 3.5 \mathrm{~mm}$ (mean \pm standard deviation) at $33 \mathrm{~cm}$ distance and $3.3 \pm 1.0 \mathrm{~mm}$ at $13 \mathrm{~cm}$ distance, respectively. Although the shorter condition increases the accuracy, the $33 \mathrm{~cm}$-distant conditions were selected for the limits of laparoscopic procedures. Although a shift and deformation of $44.6 \mathrm{~mm}$ on average was assumed because of the combined effect of respiration and pneumoperitoneum ${ }^{15-18}$, errors of the simulation have been reported within $2 \mathrm{~cm}$ in recent studies ${ }^{7,19}$. However, the dynamic navigation technique based on an electromagnetic-tracked laparoscopic ultrasound-based navigation approach reported accurate and efficient targeting of liver tumours in a laparoscopic ablation as the median target-positioning error was $4.2 \mathrm{~mm}$ and median effort time was $39 \mathrm{sec}^{20}$. The combined staining methods with contrast-enhancement ultrasonography and PDE were performed in this study and were considered feasible to improve the accuracy of the authentic plane ${ }^{6}$. Our registration procedures to section VIII, with a high degree of difficulties in laparoscopic sectionectomy, was performed just after dividing the surrounding ligaments. In addition, the procedures while holding the breath minimized errors caused by non-rigid registration of hepatic deformation or respiratory movements.

This novel navigation system for laparoscopic procedures may be feasible with acceptable accuracy within $10 \mathrm{~mm}$ errors in mean $(0-22 \mathrm{~mm})$, although further investigation, such as elevating the magnetic power and/ or additional port to use the shorter navigational instrument, is recommended to minimise the error range and perform more accurate hepatic resections. 


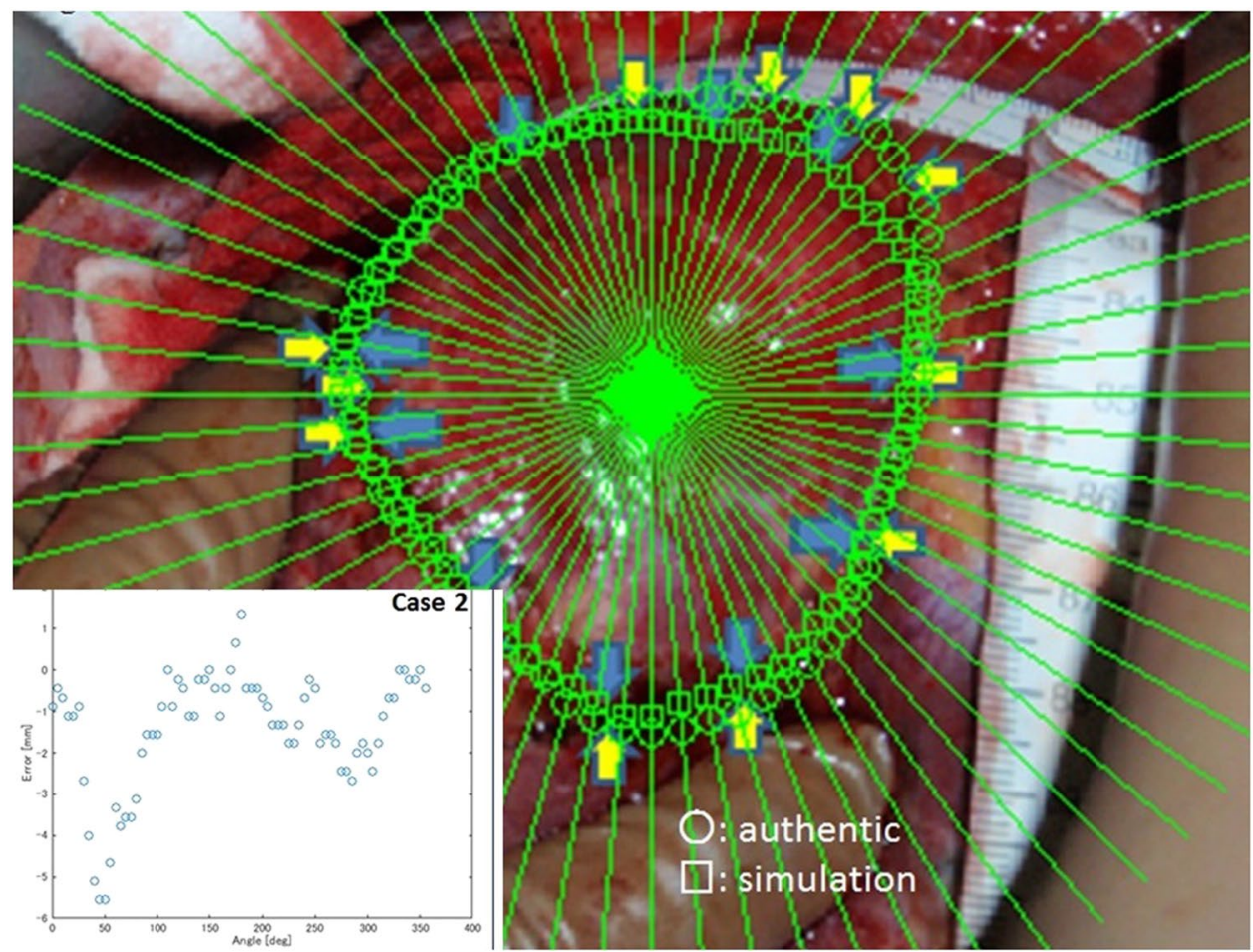

Figure 3. Errors of the dissection plane between the simulation and the authentic plane. The differences between the simulation plane and the authentic dissecting plane at every five degrees from the centre of the real excision plane were measured. The figure showed the errors found in Case 2.

\section{Conclusion}

The feasibility and safety of the navigation system for laparoscopic hepatic surgery was investigated. Errors of the simulated domain were first reported, although errors of registration points have been reported in the literatures. Although some misalignment occurred, it might be considered acceptable in the selected situation. The system should be helpful for laparoscopic navigation and liver resection procedures, although further investigation is recommended.

Ethical approval and informed consent. This clinical observation study was approved by the Institutional Review Board of JCHO Shimonoseki Medical Centre and the ethics committee group of Hitachi (\#201-1). The declaration was expressed on the title page. Written informed consent was obtained from all patients for the use of the navigation system and their clinical information.

\section{Data availability}

We declare that the data can be disclosed when requested. Registration numbers (UMIN-CTR ID): UMIN000034341 Date: 1/10/2018

Received: 22 October 2018; Accepted: 23 March 2020;

Published online: 10 April 2020

\section{References}

1. Satou, S. et al. Initial experience of intraoperative three-dimensional navigation for liver resection using real-time virtual sonography. Surgery. 155, 255-262 (2014).

2. Mise, Y. et al. Virtual liver resection: computer-assisted operation planning using a three-dimensional liver representation. J Hepatobiliary Pancreat Sci. 20, 157-164 (2013).

3. Lin, N. C., Nitta, H. \& Wakabayashi, G. Laparoscopic major hepatectomy: a systematic literature review and comparison of 3 techniques. Ann Surg. 257, 205-213 (2013).

4. Cho, A. et al. Safe and feasible extrahepatic Glissonean access in laparoscopic anatomical liver resection. Surg Endosc. 25, 1333-1336 (2011).

5. Makuuchi, M., Hasegawa, H. \& Yamazaki, S. Ultrasonically guided subsegmentectomy. Surg Gynecol Obstet. 161, 346-350 (1985).

6. Uchiyama, K. et al. Combined intraoperative use of contrast-enhanced ultrasonography imaging using a sonazoid and fluorescence navigation system with indocyanine green during anatomical hepatectomy. Langenbecks Arch Surg. 396, 1101-1107 (2011).

7. Takamoto, T. et al. Feasibility of Intraoperative Navigation for Liver Resection Using Real-time Virtual Sonography With Novel Automatic Registration System. World J Surg. 42, 841-848 (2018).

8. Yamada, T. et al. Comparison of Adverse Events and Outcomes Between Patients With and Without Drain Insertion After Hepatectomy: A Propensity Score-Matched, Multicenter, Prospective Observational Cohort Study in Japan (CSGO-HBP-001). World J Surg. 23, 4461-4469 (2018).

9. Yamamoto, M., Katagiri, S., Ariizumi, S., Kotera, Y. \& Takahashi, Y. Glissonean pedicle transection method for liver surgery (with video). J Hepatobiliary Pancreat Sci. 19, 3-8 (2012). 
10. Zhou, Y., Xu, D., Wu, L. \& Li, B. Meta-analysis of anatomic resection versus nonanatomic resection for hepatocellular carcinoma. Langenbecks Arch Surg. 396, 1109-17 (2011).

11. Ueno, S. et al. Preoperative segmentation of the liver, based on 3D CT images, facilitates laparoscopic anatomic hepatic resection for small nodular hepatocellular carcinoma in patients with cirrhosis. Hepatogastroenterology. 57, 807-812 (2010).

12. Hallet, J., Gayet, B., Tsung, A., Wakabayashi, G. \& Pessaux, P. II International Consensus Conference on Laparoscopic Liver Resection Group. Systematic review of the use of pre-operative simulation and navigation for hepatectomy: current status and future perspectives. J Hepatobiliary Pancreat Sci. 22, 353-362 (2015).

13. Nishino, H. et al. Real-time Navigation for Liver Surgery Using Projection Mapping With Indocyanine Green Fluorescence: Development of the Novel Medical Imaging Projection System. Ann Surg. 267, 1134, https://doi.org/10.1097/SLA.0000000000002172 (2018).

14. Shindoh, J. et al. Continuous ultrasound navigation for safe and precise anatomic resection of the liver. Hepato-Gastroenterology. 60, 590-594 (2013).

15. Vijayan, S. et al. Liver deformation in an animal model due to pneumoperitoneum assessed by a vessel-based deformable registration. Minim Invasive Ther Allied Technol. 23, 279, https://doi.org/10.3109/13645706.2014.914955 (2014).

16. K. Kim, et al. Changes of diaphragmatic excursion and lung compliance during major laparoscopic pelvic surgery: A prospective observational study. PLOS ONE $\mid 29,10.1371 /(2018)$

17. Galmén, K., Freedman, J., Toporek, G., Goździk, W. \& Harbut, P. Clinical application of high frequency jet ventilation in stereotactic liver ablations - a methodological study. F1000Research. 10, 1-11 (2018).

18. Dawda, S. et al. Patient-Specific Simulation of Pneumoperitoneum for Laparoscopic Surgical Planning. Journal of Medical Systems. 43, 317, https://doi.org/10.1007/s10916-019-1441-z (2019).

19. Lv A., Li Y., Qian H. G, Qiu H., Hao C. Y. Precise Navigation of the Surgical Plane with Intraoperative Real-time Virtual Sonography and 3D Simulation in Liver Resection. J Gastrointest Surg. 22, 1814-1818; 10.1007/s11605-018-3872-0. (Epub 2018 23).

20. Paolucci, I. et al. Design and implementation of an electromagnetic ultrasound-based navigation technique for laparoscopic ablation of liver tumors. Surg Endosc. 32, 3410, https://doi.org/10.1007/s00464-018-6088-1 (2018).

\section{Author contributions}

Study conception and design: Koichiro Sakata. Data acquisition: Taiki Kijima and Osamu Arai. Data analysis and interpretation: Koichiro Sakata and Osamu Arai. Drafting of the manuscript: Koichiro Sakata and Osamu Arai. Critical revision of the manuscript: Koichiro Sakata.

\section{Competing interests}

The authors declare no competing interests.

\section{Additional information}

Supplementary information is available for this paper at https://doi.org/10.1038/s41598-020-63131-3.

Correspondence and requests for materials should be addressed to K.S.

Reprints and permissions information is available at www.nature.com/reprints.

Publisher's note Springer Nature remains neutral with regard to jurisdictional claims in published maps and institutional affiliations.

(c) (i) Open Access This article is licensed under a Creative Commons Attribution 4.0 International

License, which permits use, sharing, adaptation, distribution and reproduction in any medium or format, as long as you give appropriate credit to the original author(s) and the source, provide a link to the Creative Commons license, and indicate if changes were made. The images or other third party material in this article are included in the article's Creative Commons license, unless indicated otherwise in a credit line to the material. If material is not included in the article's Creative Commons license and your intended use is not permitted by statutory regulation or exceeds the permitted use, you will need to obtain permission directly from the copyright holder. To view a copy of this license, visit http://creativecommons.org/licenses/by/4.0/.

(C) The Author(s) 2020 